\title{
Numerical Investigation of a 2D-Grating for Light Extraction of a Bottom Emitting OLED
}

\author{
Peter Vandersteegen*, Student Member, IEEE, Carl Van Buggenhout, Peter Bienstman, Member, IEEE \\ and Roel Baets, Senior Member IEEE \\ Department of Information Technology, UGent-IMEC, St-Pietersnieuwstraat 41, 9000 Gent, Belgium \\ Tel: (+32-9)264 3447, Fax: (+32-9) 264 3593, e-mail: Peter.Vandersteegen@intec.UGent.be
}

\begin{abstract}
An important limiting factor for efficient white light emitting organic LEDs is the total internal reflection occurring at each interface. In a bottom emitting OLED light is trapped by reflection at the interface between the organic layers and glass substrate and at the interface between the glass substrate and air. We investigate the use of a grating at the glass substrate-air interface. In this paper we will discuss the developed 3D-simulation method and several important simulation results. Our simulation method shows that the grating extracts approximately 50\% more power in comparison with a planar device. These results are comparable with the use of micro lenses.
\end{abstract}

Keywords: extraction efficiency, organic LED, bottom emitting, Bragg grating.

\section{INTRODUCTION}

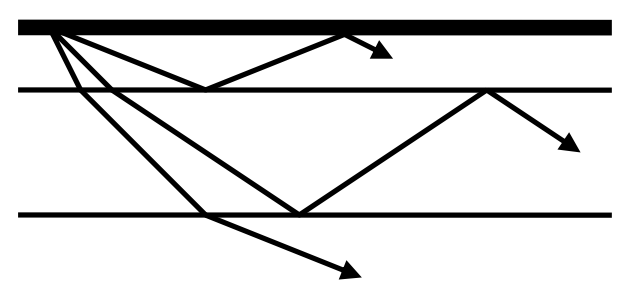

(a)

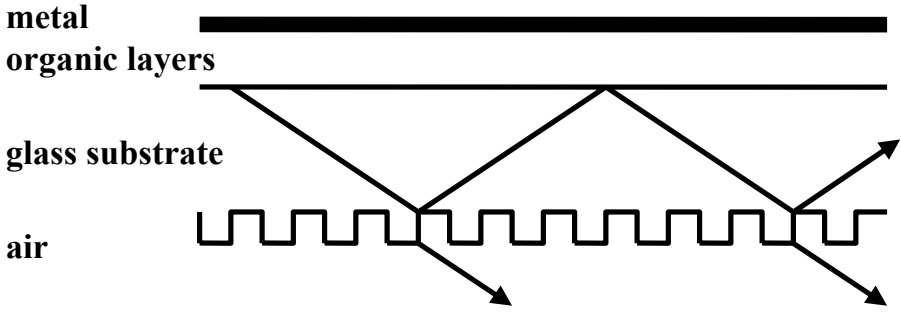

(b)

Figure 1. Extraction of light which is trapped for a planar structure.

Organic LEDs (OLEDs) are a promising technology for future lighting [1]. A key requirement for these planar lighting devices is having high brightness at reasonable efficiency or equivalently put, reasonable luminous efficacy. Increasing this efficiency is achieved by maximizing the amount of created excitons, by maximizing the radiative decay of these excitons into photons and by maximizing the amount of light which can escape an OLED device, [2]. Most of these created photons are trapped in the oLED by total internal reflections (TIR). Taking into account the refractive indices of organic layers $(\mathrm{n} \approx 1.7)$, glass $(\mathrm{n} \approx 1.5)$ and air $(\mathrm{n} \approx 1.0)$, we see that TIR occurs at the interface organic layers-glass substrate and glass substrate air, figure 1.a.

Deposition of the organic layers on a corrugated substrate has been proven to increase the efficiency for a photoluminiscent (PL) OLED [3]. Using a periodic grating at the organic layer-glass substrate interface increases out coupling for both electroluminescent (EL) and PL devices, [4] and [5]. Numerical calculations indicate an intermediate layer between organic layers and glass substrate can also improve efficiency [6]. Experimentally it has been proven that an aerogel intermediate layer increases efficiency for EL devices [7]. To eliminate the total internal reflection occurring at the glass substrate-air interface the use of microlenses [8] and a diffusive layer has been investigated [9]. Methods at the glass substrate-air interface are unable to extract light trapped in the organic layers. These methods still increase the extracted light with an additional $50 \%$ compared to a planar device.

Our alternative to these last 2 methods is the use of a grating at the glass air interface. The period of this grating is of the same order as the wavelength. The advantage of this method compared to micro lenses is the much smaller size of the grating features.

The following sections will discuss simulation method, simulation results and the conclusion.

The work presented in this paper has been (partially) funded by the European Comission under contract IST004607 (OLLA). 


\section{SIMULATION METHOD}

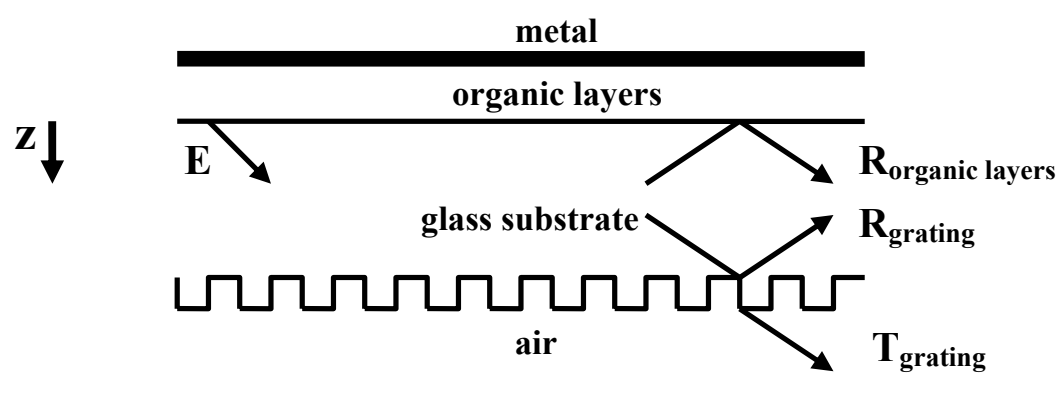

Figure 2. Matrix description of the power flux in z-direction.

With our method we determine the efficiency of a grating placed at the glass-air interface. An extraction efficiency of $100 \%$ corresponds with an extraction of $100 \%$ of all the light which is injected into the glass substrate. All light injected in the glass layer escapes into air.

The efficiency is defined as the net power flux in air proportional to the net power flux in the glass substrate. The power flux is the power flux through an elementary surface perpendicular to the z-direction.

Our model takes into account following effects: the angular distribution of both emission and absorption of the organic layers, the multiple Bragg diffraction orders and the spatial incoherent multiple reflections of figure 1.b. Spatial incoherence is assumed because the glass is thick compared to the coherence length of spontaneous emitted light.

A key element of this method is the decomposition of the radiating dipole into plane waves. This plane wave expansion calculates the electromagnetic field in the micro cavity composed by the organic layers [10]. The emitted power flux, $\mathbf{E}$, and power reflectance of the cavity organic layers, $\mathbf{R}_{\text {organic layrers, }}$, are a direct result of this calculation. Each of the resulting plane waves is propagated through the OLED structure to find the required efficiency. We have to take into account the power flux of all reflected and transmitted plane waves at each interface. Using the power flux automatically ensures the spatial incoherence of our model.

The effect of the grating on the reflected and transmitted Bragg orders can be calculated with Rigorous Coupled Wave Analysis as used in [11]. The wave vector of the plane wave incident on the grating is $\left(k_{x, 0}, k_{y, 0}, k_{z, 0}\right)$. The wave vector of all Bragg orders satisfy:

$$
\begin{aligned}
& k_{i, j}=\left(k_{x, i}, k_{y, j}, k_{z, i}\right)=\left(k_{x 0}+i k_{\Delta, x}, k_{y 0}+j k_{\Delta, y}, \sqrt{k^{2}-k_{x, i}^{2}-k_{y, i}^{2}}\right) \\
& i, j \in \mathrm{Z}=\{\ldots,-2,-1,0,1,2, \ldots\} \\
& k_{\Delta, x}=2 \pi / x \text {-period }, k_{\Delta, y}=2 \pi / y \text {-period }
\end{aligned}
$$

Only diffraction orders with a real $k_{z, i}$ are plane waves with a non-zero power flux. All plane waves which satisfy equation (1) for a certain $\left(k_{x, 0}, k_{y, 0}\right)$ form a set. We can place the reflection of the diffraction orders for any incident plane wave which satisfies (1) in one of the column of the square matrix $\mathbf{R}_{\text {grating. }}$. The power transmission of the flux can be written in an $\mathrm{n}$ dimensional array $\mathbf{T}_{\text {grating. }}$

For any set of power carrying Bragg orders we write.

$$
\begin{gathered}
\text { total flux for one set }=\mathbf{T}_{\text {grating. }} \mathbf{E}+\mathbf{T}_{\text {grating. }} \mathbf{R}_{\text {organic layers }} \mathbf{R}_{\text {grating }} \mathbf{E}+\mathbf{T}_{\text {grating. }}\left(\mathbf{R}_{\text {organic layers }} \mathbf{R}_{\text {grating }}\right)^{2} \mathbf{E}+\ldots \\
=\mathbf{T}_{\text {grating. }}\left(\mathbf{1}-\mathbf{R}_{\text {organic layers }} \mathbf{R}_{\text {grating }}\right)^{-1} \mathbf{E}
\end{gathered}
$$

The final field can be found by integrating over all plane waves. 


\section{SIMULATION RESULTS}

\begin{tabular}{|c|c|}
\hline AlQ3 & $: 30 \mathrm{~nm}, \quad \mathrm{n}=1.655$ \\
\hline$\alpha-N P D$ & $: 30 \mathrm{~nm}, \quad \mathrm{n}=1.807$ \\
\hline ITO & : $120 \mathrm{~nm}, \mathrm{n}=1.806-0.012 \mathrm{j}$ \\
\hline SiON & $: 100 \mathrm{~nm}, \mathrm{n}=1.622$ \\
\hline glass & $: n=1.528$ \\
\hline
\end{tabular}

Figure 3. Organic layer stack.

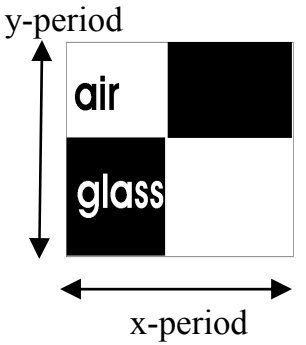

(a)

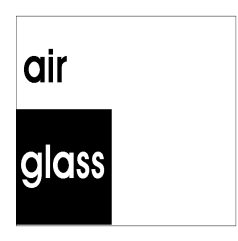

(b)

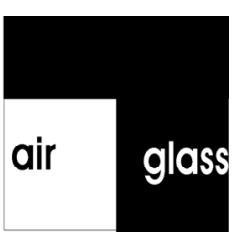

(c)

Figure 4. Top view of one period of the grating.

The used OLED layer stack is shown in Figure 3. This stack has been used to calculate for both emission and reflection of the organic layers. In the following discussion we make a distinction between the 3 grating types of Figure 4. In this figure we only show one period of the grating. We have investigated the influence of following parameters: period, filling factor and grating depth. For Figure 4 (a) and (b) we define the fill factor as the proportion between glass and period. The fill factor of Figure 4 (c) has been defined as the proportion between air and period. Although both the period and the filling factor can be varied independently in $\mathrm{x}$ and $\mathrm{y}$ direction, we always have used the same value in $\mathrm{x}$ and $\mathrm{y}$ direction. Unless indicated otherwise the used grating has a depth of $500 \mathrm{~nm}$, a filling factor of 0.65 and a period of $1.0 \mu \mathrm{m}$.

We have found that a grating increases efficiency with approximately $50 \%$ compared to a planar interface. This is comparable with micro lenses. The distinction between 3 grating types only has implications for the determination of the optimal fraction.

\subsection{Period}

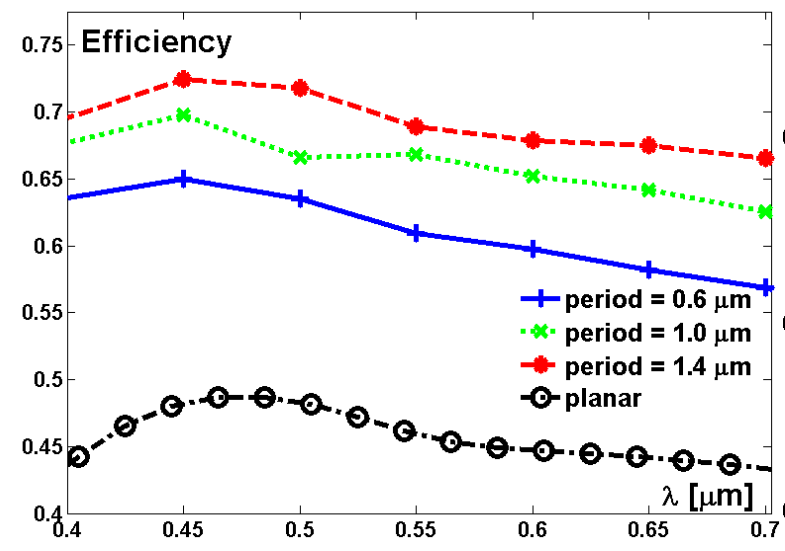

Figure 5. Efficiency in function of the wavelength for several grating Periods.

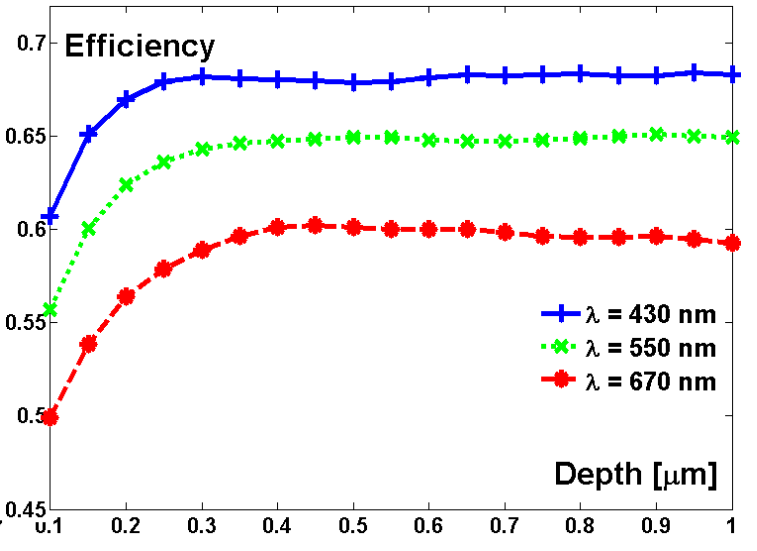

Figure 6. Efficiency in function of the Depth.

Figure 5 gives an indication of the efficiency boost achieved by using a grating based on Figure 4 (a). Using a planar interface at the glass-air interface we have calculated that approximately $45 \%$ of the light is extracted to air. Using a grating on top of the glass, we see an efficiency of approximately $70 \%$. Figure 5 shows an increase of efficiency for larger periods. This improvement for larger periods stops for periods larger than $1.5 \mu \mathrm{s}$. It should be noted that similar results were achieved by using grating types from figure Figure 4 (b) and (c).

\subsection{Depth}

Figure 6 shows the increase for efficiency for several wavelengths. A maximum efficiency is achieved for each wavelength. Longer wavelengths require more deep gratings. Taking into account the maximum wavelength of the visible spectrum a depth of $500 \mathrm{~nm}$ should be sufficient to maximize out coupling.

Similar results were achieved by using grating types from figure Figure 4 (b) and (c). 


\subsection{Fill factor}

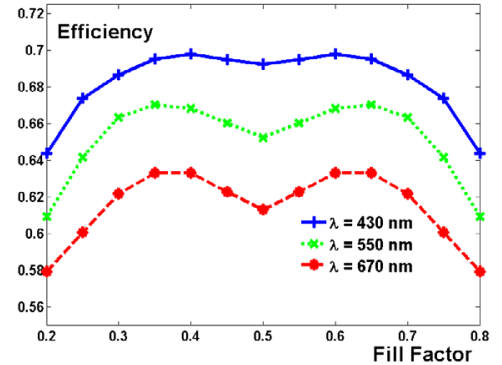

(a)

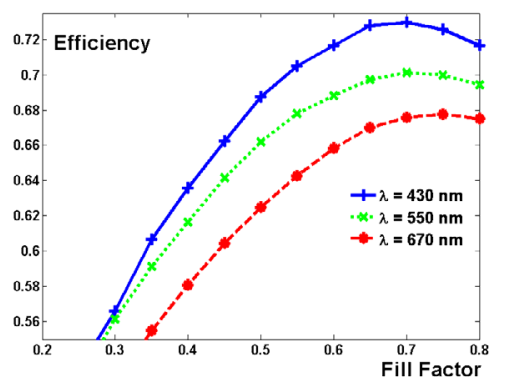

(b)

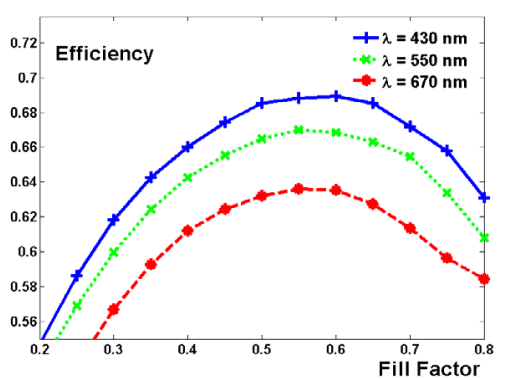

(c)

Figure 7. Efficiency in function of fill factor for several wavelengths. Each graph (a,b or c) corresponds with the grating type described in Figure 4.

Figure 7 shows the influence of the fill factor on the different grating types of Figure 4. Each structure type has its own optimum. For a certain structure type, the optimal filling factor is the same for all wavelengths.

\section{CONCLUSIONS}

We have developed a simulation tool for gratings at the glass air interface for a bottom emitting OLED. Several parameters for different gratings have been investigated. A minimal depth is required for optimal efficiency. The efficiency increases for larger grating periods. This efficiency increase stops for grating periods bigger than $1.5 \mu \mathrm{m}$. We get an increase of $50 \%$ for the extraction efficiency compared to a planar structure. This is comparable with micro lenses. Future numerical simulation should give insight in the angular distribution of the emitted field. We plan to fabricate these gratings with interference lithography.

\section{ACKNOWLEDGEMENTS}

We thank the European Commission for funding of (part of) this work under contract IST-004607 (OLLA).

\section{REFERENCES}

[1] Organic LEDs Look Forward to a Bright, "White Future," Science (Magazine), vol. 310, no.5755, pp. 1762-1763. December 2005.

[2] N. K. Patel, et al., "High-Efficiency Organic Light-Emitting Diodes," IEEE Journal on Selected Topics in Quantum Electronics, vol. 8, no. 2, pp. 364-361, March/April 2002.

[3] Benjamin Matterson, et al., "Increased Efficiency and Controlled Light Output from a Microstructured Light-Emitting Diode," Advanced Materials, vol. 13, no. 2, pp. 123-127, January 2001.

[4] Jonathan M. Ziebarth, et al., "Extracting Light from Polymer Light-Emitting Diodes Using Stamped Bragg Gratings," Advanced Functional Materials, vol. 14, no. 5, pp. 451-456.May 2004.

[5] Masatoshi Kitamura, et al., "Enhanced Luminance Efficiency of Organic Light-Emitting Diodes with Two-Dimensional Photonic Crystals," Japanese Journal of Applied Physics, vol. 44, no.4B, pp. 28442848, 2005.

[6] Kristiaan Neyts, "Microcavity Effects an the outcoupling of light in displays and lighting applications based on thin emitting films," Applied Surface Science, vol. 244, pp. 4517-523, 2005.

[7] Tetsuo Tsutsui, et al., "Doubling Coupling-out Efficiency in Organic Light-Emitting Devices Using a Thin Silica Aerogel Layer," Advanced Materials, vol. 13, no. 15, pp. 1149-1152, August 2001.

[8] S. Möller, et al., "Improved light out-coupling in organic light emitting diodes employing ordered microlens arrays," Journal of Applied Physics, vol. 91, no. 5, pp. 3324-3327, March 2002.

[9] Toshitaka Nakamura, et al., "Improvement of coupling-out efficiency in organic electroluminescent devices by addition of a diffusive layer," Journal of Applied Physics, vol. 96, no.11, pp. 6016-6022, December 2004.

[10] CAMFR simulation software is freely available from http://camfr.sourceforge.net

[11] Danaë Delbeke, et al., "Rigorous electromagnetic analysis of dipole emission in periodically corrugated layers: the grating assisted resonant-cavity light-emitting diode," Journal of the Optical Society of America $A$, vol.19, no. 5, May 2002. 


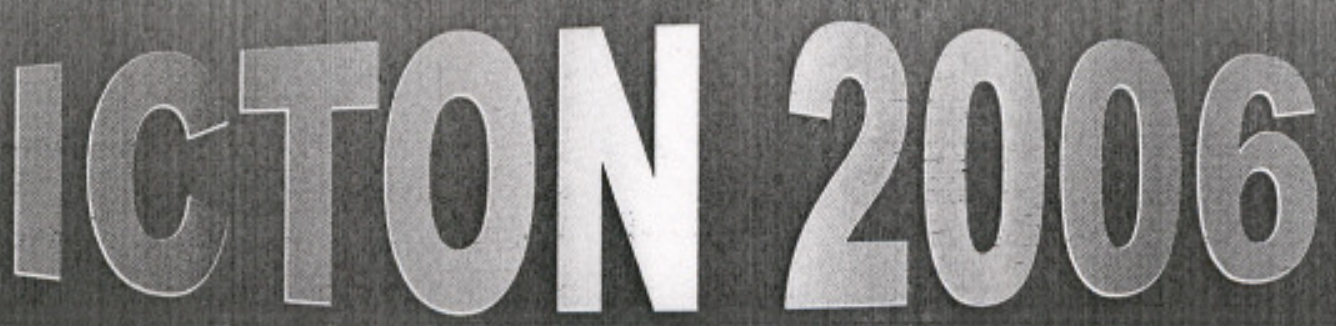

\title{
$8^{\text {th }}$ International Conference
}

on

\section{Transparent Optical Networks}

co-located with:

\begin{abstract}
ESPC $\quad 5^{\text {n }}$ European Symposium on Photonic Crystals
WAOR $\quad 5^{\text {n }}$ Workshop on All-Optical Routing

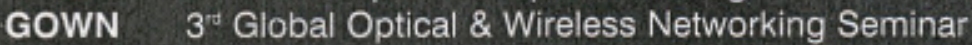

RONEXT $2^{\text {r }}$ COST 270 Workshop on Reliability Issues in Next Generation Optical Networks

PICAW $\quad 2^{\text {rd }}$ Photonic Integrated Components \& Applications Workshop

NAON COST 288 Nanophotonics for All-Optical Networking Workshop

GRAAL COST 293 Annual Conference on Graphs and Algorithms in Communication Networks

COST P11 Training School: Modelling and Simulation Tecinniques for Linear, Nonlinear and Active

Photonic Crystals

\section{and special sessions:}

MPM - Microresonators and Photonic Molecules: trapping, harnessing and releasing light Industrial
\end{abstract}

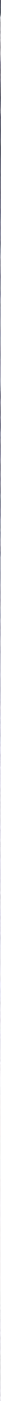


Editor: Marian Marciniak

Associate Editor: Marek Jaworski

Technical Editors: Marcin Chochot, Mariusz Zdanowicz

Cover photos by Hanna Skrobek.

front - panoramic view of the University park campus with Trent building

back - Nottingham castle wall with Robin Hood statue

Printed by National Institute of Telecommunications, Transmission and Fiber Optics Department

Copyright and Reprint Permission: Abstracting is permitted with credit to the source. Libraries are permitted to photocopy beyond the limit of U.S. copyright law for private use of patrons those articles in this volume that carry a code at the bottom of the first page, provided the per-copy fee indicated in the code is paid through Copyright Clearance Center, 222 Rosewood Drive, Danvers, MA 01923. For other copying, reprint or republication permission, write to IEEE Copyrights Manager, IEEE Operations Center, 445 Hoes Lane, P.O. Box 1331, Piscataway, NJ 088551331. All rights reserved. Copyright $\odot 2006$ by the Institute of Electrical and Electronics Engineers, Inc.

IEEE Catalog Number: 06EX1326

ISBN: 1-4244-0235-2

Library of Congress: 2006921097 